\title{
Tratamento cirúrgico da insuficiência mitral em crianças: dez anos de técnicas reparadoras
}

\author{
Francisco GREGORI Jr.", Samuel S. SILVA ${ }^{\star \star}$, Osney MOURE ${ }^{\star \star \star}$, Roberto TAKEDA ${ }^{\star * \star *}$, Luciano FAÇA- \\ NHA $^{* * *}$, Icanor RIBEIRO***, Luiz GÓIS ${ }^{\star * * *}$, José SIQUEIRA*, Thelma F. GREGORI ${ }^{\star \star * \star *}$, Ascêncio G. LO- \\ PES ${ }^{* * * \star}$, Pedro KRELING**, Luiz C. MIGUITA****, Celso CORDEIRO****
}

RBCCV 44205-94
GREGORI Jr., F.; SILVA, S. S.; MOURE, O.; TAKEDA, R.; FAÇANHA, L.; RIBEIRO, I.; GÓIS, L.; SIQUEIRA, J.; GREGORI, T. F.; LOPES, A. G.; KRELING, P.; MIGUITA, L. C.; CORDEIRO, C. - Tratamento cirúrgico da insuficiência mitral em crianças: dez anos de técnicas reparadoras. Rev. Bras. Cir. Cardio- vasc., 4(3): 202-209, 1989.

RESUMO: Entre abril de 1979 e janeiro de 1989, de 71 pacientes com idade entre 4 e 16 anos (média $11,5)$ portadores e insuficiência mitral (IM), $53(74,6 \%)$ foram submetidos s procedimentos plásticos do aparelho valvar mitral, dos quais $29 \mathrm{com}$ menos de 12 anos. Todos apresentavam IM, 21 deles com estenose mitral associada (dupla lesão mitral - DLM). Em $21(39,6 \%)$ pacientes, foi observada regurgitação tricúspide à cineventriculografia direita. A etiologia reumática foi predominante (42 casos, $79,2 \%$ ). Todos os pacientes, exceto três situavam-se na classe funcional III e IV. Foram empregados anéis de Carpentier em 41 pacientes e, nos últimos 10, anéis abertos Gregori-IMC, associados a procedimentos plásticos sobre as cúspides e o aparelho subvalvar. Em dois pacientes, a plastia mitral fo realizada sem o emprego do anel protético. Não ocorreram óbitos hospitalares. Três $(5,7 \%)$ pacientes foram reoperados para troca valvar, todos pertencentes ao grupo com estenose mitral associada quando 1 faleceu, sendo o único óbito tardio de toda a série $(1,9 \%)$. Na avaliação clínica, com tempo pós-operatório médio de 5 anos (1 mês a 10 anos), 2 $(3,8 \%)$ estavam sem seguimento, $43(81,1 \%)$ na classe funcional I, $4(7,5 \%)$ na classe funcional II e 4 $(7,5 \%)$ na classe III. Dos trēs pacientes pertencentes à classe II no pós-operatório, todos evoluíram para a classe I. Dos 26 da classe III, $23(88,5 \%)$ passaram para a classe I e 1 permaneceu na III. Dos 24 pacientes da classe IV, $15(62,5 \%)$ passaram para a I, 4 passaram para a II, 3 passaram para a classe III e 2 sem seguimento. Vinte e dois pacientes $(41,5 \%)$ encontravam-se sem sopros, $15(28,3 \%)$ com sopro sistólico no foco mitral (SSFM) discreto $(+1++++), 10(18,9 \%)$ com SSFM moderado $(++1++++)$, $4(7,5 \%)$ com SSFM importante $(+++1++++), 2(3,8 \%)$ sem seguimento. Dos 3 pacientes com IM pura, $30(93,8 \%)$ estavam na classe I, $1(3,1)$ na classe II e $1(3,1 \%)$ sem seguimento. Neste grupo, $16(50 \%)$ pacientes encontravam-se sem sopros, $9(28,1 \%)$ com SSFM discreto, $6(18,9 \%)$ com SSFM moderado e $1(3,1 \%)$ sem seguimento. Dos 21 pacientes com estenose mitral associada $13(61,9 \%)$ situavam-se na classe funcional I, $4(19 \%)$ na classe II, $3(14,3 \%)$ na classe III e $1(4,8 \%)$ sem seguimento. Neste grupo, $6(28,6 \%)$ encontravam-se sem sopros, $6(28,6 \%)$ com SSFM discreto, $4(19 \%)$ com SSFM moderado, $4(19 \%)$ com SSFM importante e $1(4,8 \%)$ sem seguimento. O reestudo hemodinâmico foi realizado em média após dois anos (um mês a sete anos) em 32 pacientes $(60,4 \%)$. Houve diminuição significativa da pressāo sistólica em artéria pulmonar $(55,4-41,9 \mathrm{P}<0,01)$, da pressão capilar pulmonar $(26,0-19,6 \mathrm{P}<0,01)$ e do gradiente transvalvar mitral $(9,6-5,7 \mathrm{P}<0,01)$, sndo que em apenas

Trabalho realizado na Faculdade de Medicina da Universidade de Londrina, no Hospital Evangélico e na Santa Casa de Londrina. Londrina, Pr, Brasil.

Apresentado ao 16: Congresso Nacional de Cirurgia Cardiaca. São Paulo, SP, 7 e 8 de abril e 1989.

- Da Faculdade de Medicina da Universidade de Londrina, do Hospital Evangélico e da Santa Casa de Londrina

.. Da Faculdade de Medicina da Universidade de Londrina e do Hospital Evangélico.

... Do Hospital Evangélico.

.... Do Hospital Evangélico e da Santa Casa de Londrina.

..... Da Faculdade de Medicina da Universidade de Londrina.

Endereço para separatas: Francisco Gregori Jr. Av. Bandeirantes, 994. 86015 Londrina, PR. 
GREGORI Jr. F.; SILVA, S. S.; MOURE, O.; TAKEDA, R.; FAÇANHA, L.; RIBEIRO, I.; GOIS, L.; SIQUEIRA, J.; GREGORI, T. F.; LOPES, A. G.; KRELING, P.; MIGUITA, L. C.; CORDEIRO, C. - Tratamento cirúrgico da insuficiência mitral em crianças: dez anos de técnicas reparadoras. Rev. Bras. Cir. Cardiovasc., 4(3): 202-209, 1989.

\begin{abstract}
$6(20 \%)$ este era superior a $7 \mathrm{~mm} / \mathrm{Hg}$ (3 deles correspondem aos casos reoperados). Vinte e três $(71,9 \%)$ pacientes apresentaram funcionalidade valvar bastante satisfatória, sendo 16 deles em regurgitação e 7 com regurgitação discreta; em 6 pacientes $(18,8 \%)$, a regurgitaçăo era moderada e em $3(9,4 \%)$ havia regurgitação importante (casos reoperados). No grupo de pacientes com IM pura (17), em $14(82,4 \%)$ a regurgitaçāo era ausente ou discreta e em $3(17,6 \%)$ era moderada. No grupo com DLM (15), em 9 $(60 \%)$ a regurgitação era ausente ou discreta, em $3(20 \%)$ era moderada e em $3(20 \%)$, importante. Dos 2 pacientes sem anel protético, um não apresentou refluxo valvar mitral e outro, moderado. No grupo de pacientes com anel de Carpentier (22), 9 estavam sem refluxo valvar, 6 com refluxo discreto, 4 moderado e 3 importante. No grupo com anel Gregori-IMC (8), 6 nāo apresentavam refluxo mitral, 1 tinha refluxo discreto e 1 , refluxo moderado.
\end{abstract}

DESCRITORES: valva mitral, cirurgia, crianças.

\section{INTRODUÇÃO}

A incidência de complicações com o emprego de próteses valvares têm estimulado os cirurgiōes a adotarem técnicas reparadoras para o tratamento cirúrgico da insuficiência mitral (IM).

Entretanto, técnicas de plicatura do anel ou, mesmo, anuloplastias isoladas não têm aprsentado resultados satisfatórios $^{2}$. A abordagem global das lesões da valva mitral proposta por vários autores ${ }^{1,3,4,7,9,10}$ propicia excelentes resultados pós-operatórios. Este fato é, marcadamente, importante quando se trata de pacientes jovens onde as lesões da valva mitral levam a manifestações clínicas precocemente e onde os resultados tardios com as substituiçōes valvares são bastante satisfatórios. Estes fatos levaram-nos, a partir de 1979, a empregar as plásticas valvares em pacientes com insuficiência mitral, especialmente crianças. $\mathrm{O}$ objetivo deste estudo é avaliar os resultados pós-operatórios em pacientes com idade inferior a 16 anos submetidos a cirurgia reparadora da valva mitral insuficiente.

\section{CASUISTICA E MÉTODOS}

De abril de 1979 a janeiro de 1989, 53 (74,6\%), de 71 pacientes operados para tratamento cirúrgico da IM, puderam ter suas valvas reparadas por técnicas cirúrgicas conservadoras. Este índice de cirurgia reparadora aumentou para $86,2 \%$ ( 25 casos), se analisarmos apenas os casos operados nos últimos dois anos (29).

A idade variou de 4 a 16 anos, com média de 11,5 anos, sendo que 29 pacientes tinham idade inferior a 12 anos. Dezessete pacientes eram do sexo masculino e 36 do feminino.

O diagnóstico pré-operatório de IM pura foi confirmado em 3 pacientes e de dupla lesão mitral em 21. Em 21 pacientes $(39,6 \%)$, foi observada regurgitaçāo tricúspide à cineventriculografia direita, apesar de, clinicamente, o diagnóstico de insuficiēncia tricúspide ter sido confirmado em apenas 12. Dois pacientes apresentavam comunicação interatrial e dois, insuficiência aórtica asso- ciada. Quarenta e dois pacientes $(79,2 \%)$ tinham passado reumático positivo, ou o aspecto intra-operatório sugeria, fortemente, tratar-se de lesão mitral reumática, estando dois na fase ativa da doença. Em cinco pacientes, a etiologia era congênita, em um era de endocardite infecciosa e, no restante, (6) a etiologia era indeterminada. Três pacientes encontravam-se no estagio II da classificação de insuficiência cardiaca (NYHA), 26 no estádio III e 24 no estádio IV. Os pacientes no estádio II apresentavam importante aumento da área cardíaca no estudo radiográfico do tórax. Sopro sistólico no foco mitral $++1++++$ foi observado em cinco pacientes, $+++1++++$ em $24++++1++++$ em 24 . Todos foram submetidos a cateterismo cardíaco no pré-operatório e a cirurgia indicada foi baseada nestes resultados.

A cirurgia foi realizada com auxílio de circulação extra-corpórea (CEC) e hipotermia sistêmica moderada com anóxia intermitente do miocárdio. Exceto em dois pacientes em que se aplicou plicatura de Woole (WOOLER et alii ${ }^{13}$ ), as técnicas de plastia valvar mitral consistiram na anuloplastia com anel de Carpentier (CARPENTIER $^{4}$ ) em 41 casos e com anel aberto Gregory-IMC (GREGORY Jr. et alii ${ }^{6}$ ) nos últimos 10 casos, acompanhados dos seguintes procedimentos ${ }^{4,} 7$ : comissurotomia, papilotomia, encurtamento de cordas alongadas, secçōes de cordas retráteis, feitura de nova corda, transposição de cordas, fechamento de orifício na cúspide anterior e ressecçōes parciais da cúspide anterior e posterior. O tempo de circulação extracorpórea foi de 75 minutos (32-122) e anóxia do miocárdio de 42 (14-78). Dois pacientes tiveram defeito do septo interatrial fechado e dois tiveram a valva aórtica substituída por próteses mecânicas.

Todos os pacientes, exceto dois, foram reestudados clinicamente no pós-operatório, com tempo médio de 5 anos ( 1 mês a 10 anos). Trinta e dois pacientes $(60,4 \%)$ foram reestudados hemodinamicamente, com tempo médio de pós-operatório de dois anos (1 mês a 7 anos).

Foi aplicada a curva atuarial de Kaplan-Mayer (SAUAIA ${ }^{11}$ ) para análise da sobrevida tardia dos pacientes e também dos pacientes livres de substituição valvar. 
GREGORI Jr. F.; SILVA, S. S.; MOURE, O.; TAKEDA, R.; FAÇANHA, L.; RIBEIRO, I.; GÓIS, L.; SIQUEIRA, J.; GREGORI, T. F.; LOPES, A. G.; KRELING, P.; MIGUITA, L. C.; CORDEIRO, C. - Tratamento cirúrgico da insuficiência mitral em crianças: dez anos de técnicas reparadoras. Rev. Bras. Cir. Cardiovasc., 4(3): 202-209, 1989.

Foram realizadas análises comparativas dos dados pré e pós-operatórios da pressão sistólica em artéria pulmonar, pressão capilar pulmonar e gradiente transvalvar mitral (teste T de Student para amostras pareadas), além da avaliação da regurgitação valvar mitral pela cineventriculografia esquerda em obliqua anterior direita.

\section{RESULTADOS}

Não ocorreram óbitos hospitalares.

Três $(5,7 \%)$ pacientes foram reoperados para troca valvar, todos pertencentes ao grupo com estenose mitral associada, quando um faleceu, sendo o único óbito tardio de toda a série $(1,9 \%)$.

À alta hospitalar, no grupo de pacientes operados entre 1979 e 1983 (21), havia ausência de sopros em $4(19 \%)$, sopro sistólico no foco mitral (SSFM) discreto em $9(42,9 \%)$, SSFM moderado em $5(23,8)$ e SSFM importante em $3(14,3 \%)$. Nos pacientes operados entre 194 e 1989 (32), foi observada ausência de sopros em $18(56,3 \%)$, SSFM discreto em $8(25 \%)$, SSFM moderado em $5(15,6 \%)$ e SSFM importante em $1(3,1 \%)$.

$\mathrm{Na}$ avaliação clínica tardia (Gráfico 1), 2 (3,8\%) estavam sem seguimento, $43(81,1 \%)$ estavam na classe funcional I, $4(7,5 \%)$ na classe funcional II e $4(7,5 \%)$ na classe III.

Dos três pacientes pertencentes à classe II no pré-operatório, todos evoluíram para a classe I. Dos 26 pacientes da classe III, $23(88,5 \%)$ passaram para a classe I e 1 permaneceu na III. Dos 24 pacientes da class IV, $15(62,5 \%)$ passaram para a I, 4 para a II e 3 para a classe III.

Vinte e dois pacientes $(41,5 \%)$ encontravam-se sem sopros, $15(28,3 \%)$ com SSFM discreto $(+/++++)$, $10(18,9 \%)$ com SSFM moderado $(++/++++)$ e 4 $(7,5 \%)$ com SSFM importante $(+++1++++)$.

\section{GRÁFICO 1}

EVOLUÇÃO CLINICA POSS-OPERATÓRIA DA CLASSE FUNCIONAL (NYHA)

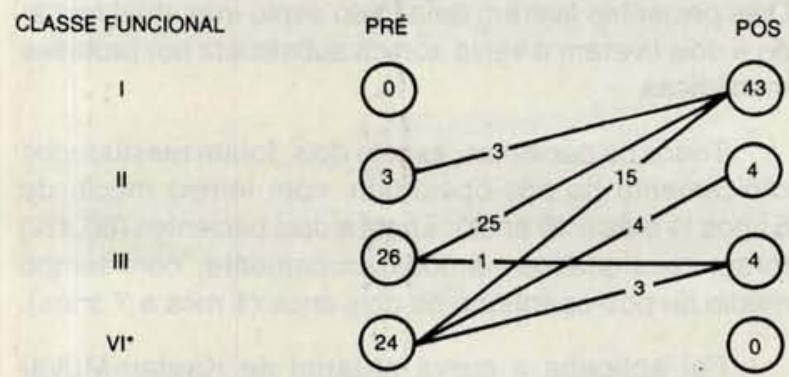

- 2 sem seguimento.
GRÁFICO 2

ANALISE ATUARIAL DOS PACIENTES SUBMETIDOS A CIRURGIA REPARADORA, QUANTO A SOBREVIDA E POSSIBILIDADE DE ESTAREM LIVRES DE REOPERACCAOO PARA SUBSTITUICAOO VALVAR MITRAL, AOS DEZ ANOS DE POS-OPERATORIO

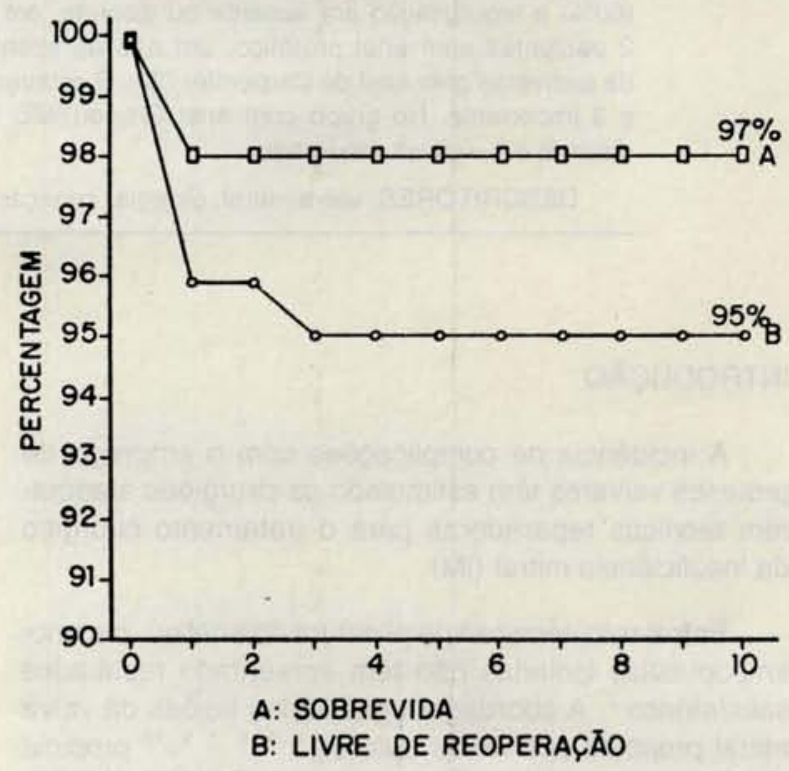

Dos trinta e dois pacientes com IM pura, $30(93,8 \%)$ estavam na classe I, $1(3,1 \%)$ na classe II e $1(3,1 \%)$ sem seguimento. Neste grupo, $16(50 \%)$ pacientes encontravam-se sem sopros, $9(28,1)$ com SSFM discreto, $6(18,9 \%)$ com SSFM moderado e $1(3,1 \%)$ sem seguimento. Dos 21 pacientes com estenose mitral associada, $13(61,9 \%)$ situavam-se na classe I, $4(19 \%)$ na classe II, $3(14,3 \%)$ na classe III e $1(4,8 \%)$ sem seguimento. Neste grupo, $6(28,6 \%)$ encontravam-se sem sopros, $6(28,6 \%)$ com SSFM discreto, $4(19 \%)$ com SSFM moderado, $4(19 \%)$ com SSFM importante e $1(4,8 \%)$ sem seguimento.

A curva atuarial ${ }^{11}$ (Gráfico 2) mostrou probabilidade de sobrevida dos pacientes aos 10 anos de $98 \%$ e de pacientes livres de reoperação para substituição valvar de $95 \%$.

O reestudo hemodinâmico foi realizado em 32 pacientes $(60,4 \%)$, sendo dois sem anel protético (tempo de seguimento 7 anos), $22 \mathrm{com}$ anel de Carpentier (tempo de seguimento 2 anos e 6 meses) e oito com anel de Gregori-IMC (tempo de seguimento 3 meses).

Houve diminuição significativa da pressão sistólica em artéria pulmonar $(55,4-41,9 \mathrm{P}<0,01)$, da pressāo capilar pulmonar $(26,0-19,6 \mathrm{P}<0,01)$ e do gradiente transvalver mitral $(9,6-5,7 \mathrm{P}<0,01)$, sendo que em apenas $6(20 \%)$ este era superior a $7 \mathrm{~mm} / \mathrm{Hg}$ (três deles correspondem aos casos reoperados). Gradiente transvalvar aórtico foi observado em dois casos com anel de Carpentier, nenhum superior a $20 \mathrm{~mm} / \mathrm{Hg}$. 
GREGORI Jr. F.; SILVA, S. S.; MOURE, O.; TAKEDA, R.; FAÇANHA, L.; RIBEIRO, I.; GÓIS, L.; SIQUEIRA, J.; GREGORI, T. F.; LOPES, A. G.; KRELING, P.; MIGUITA, L. C.; CORDEIRO, C. - Tratamento cirúrgico da insuficiência mitral em crianças: dez anos de técnicas reparadoras. Rev. Bras. Cir. Cardiovasc., 4(3): 202-209, 1989.

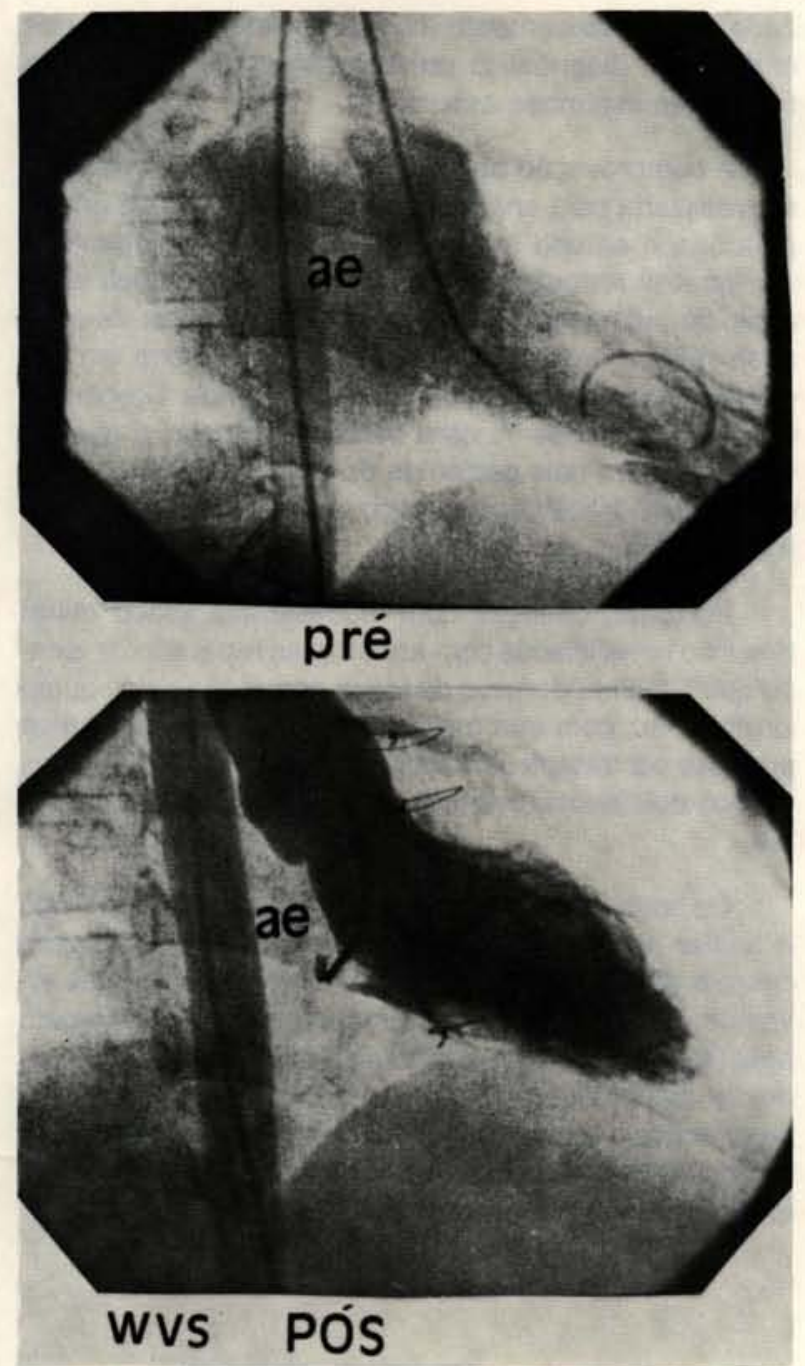

Fig. 1 - Cineventriculografia esqueda pré e pós-operatória em posiçâo obliqua anterior direita de um paciente de oito anos de idade submetido a plástica valvar mitral com interposição de anel de Gregori-IMC. Notar ausência de refluxo mitral de contraste no pós-operatório.

Vinte e três $(71,9 \%)$ pacientes apresentavam funcionalidade valvar bastante satisfatória (Figura 1), sendo 16 deles sem regurgitaçāo e sete com regurgitação discreta; em seis pacientes $(18,8 \%)$ a regurgitação era moderada e em três era importante (casos reoperados). No grupo de pacientes com IM pura (17), em 14 (82,4\%) a regurgitação estava ausente ou discreta e em 3 $(17,6 \%)$, moderada. No grupo com DLM (15), em nove (60\%) a regurgitação estava ausente ou discreta, em três $(20 \%)$ era moderada e em três $(20 \%)$ era importante.

Dos pacientes sem anel protético, um não apresentou refluxo valvar mitral e outro apresentou refluxo moderado. No grupo de pacientes com anel de Carpentier, nove estavam sem refluxo valvar, seis estavam com refluxo discreto, em quatro o refluxo era moderado e em três era importante. No grupo com anel Gregori-IMC, seis não apresentavam refluxo mitral, um apresentava refluxo discreto e um, refluxo moderado (paciente na fase ativa da doença reumática).

\section{CONCLUSÕES}

1) Uma alta percentagem de crianças com valva mitral insuficiente pode ser tratada com cirurgia reparadora.

2) As cirurgias podem ser realiadas com baixa mortalidade hospitalar,

3) A ausculta pós-operatória imediata foi melhor nos pacientes operados nos últimos cinco anos (1984-1989) em comparação com os do período anterior (1973-1983).

4) $O$ índice de reoperação é bastante aceitável no pósoperatório tardio.

5) As evoluçōes clínicas e hemodinâmicas tardias são satisfatórias.

6) Melhores resultados são observados no grupo de pacientes com classe funcional pré-operatória II e III.

7) A evolução é melhor no grupo de pacientes com insuficiência mitral sem estenose associada.

\section{COMENTÁRIOS}

Em nosso meio, as lesōes da valva mitral têm sido, mais freqüentemente, secundárias à febre reumática, principalmente em crianças e jovens onde a degeneração mixomatosa e as disfunçōes do músculo papilar isquêmico são, praticamente, ausentes.

Durante o surto da doença, não somente as valvas são acometidas, mas também o miocárdio, sendo a sua função contrátil comprometida não apenas pelas alterações hemodinãmicas secundárias à disfunção da valva mitral, como também pelo processo inflamatório que atinge as fibras musculares cardiacas. Esta combinação de fatores pode levar à deterioração da funçāo cardiaca, culminando em grande cardiomegalias. $\mathrm{O}$ tratamento cirúrgico dos pacientes com insuficiência mitral, qualquer que seja sua etiologia, resolve o problema hemodinâmico, aliviando a sobrecarga imposta ao miocárdio, porém, se este tratamento nāo se fizer em tempo adequado, as lesōes do miocárdio podem se tornar definitivas, sendo os resultados tardios bastante sombrios, fato mais comumente observado em crianças.

A cirurgia mais freqüentemente adotada nestes casos tem sido substituição do aparelho valvar mitral por válvulas artificiais.

As complicaçōes fartamente relatadas após o emprego das próteses artificiais em posição mitral, mais intensas em crianças e jovens, têm levado os cirurgiôes 
GREGORI Jr. F.; SILVA, S. S.; MOURE, O.; TAKEDA, R.; FAÇANHA, L.; RIBEIRO, I.; GOIS, L.; SIQUEIRA, J.; GREGORI, T. F.; LOPES, A. G.; KRELING, P.; MIGUITA, L. C.; CORDEIRO, C. - Tratamento cirúrgico da insuficiência mitral em crianças: dez anos de técnicas reparadoras. Rev. Bras. Cir. Cardiovasc., 4(3): 202-209, 1989.

a postergarem a cirurgia até que o quadro clínico exija, com grandes aumentos da área cardíaca e que, se por um lado este é um mau fato, por outro facilita o ato operatório, permitindo a implantação de substitutos valvares maiores.

As cirurgias reparadoras da valva mitral ganharam destaque e mostraram a superioridade de seus resultados em relação ao das trocas valvares somente após o aparecimento das técnicas propostas por CARPEN$\mathrm{TIER}^{4}$. Lesões valvares puderam ser corrigidas tornando as valvas competentes sem limitar o movimento de seus folhetos, não levando, portanto, à estenose. Pacientes de idade mais baixa puderam ser beneficiados com estas técnicas e a cirurgia, mais precocemente indicada. $\mathrm{Na}$ nossa casuística, mais de cinqüenta por cento dos pacientes tinham idade inferior a 12 anos.

Apesar de serem cirurgias mais trabalhosas, os resultados imediatos e tardios justificam sua aplicação. A maioria dos Serviços ${ }^{1,3,5,10}$ que adotam os procedimentos reparadores tem obtido mortalidade hospitalar bastante satisfatória, inferior à das trocas valvares, fato observado, também, neste estudo, já que nāo tivemos óbitos imediatos.

A incidēncia de reoperaçāo tardia para substituição valvar, descrita na literatura mundial ${ }^{1}, 5,10$, é bastante aceitável e os nossos resultados foram semelhantes $(5,7 \%)$. Mais de oitenta por cento dos nossos pacientes encontravam-se no pós-operatório em classe funcional I e com ausculta mitral satisfatória, sendo os melhores resultados observados nos pacientes com cirurgia reali- zada mais precocemente (classe funcional II e III) e nos casos com diagnóstico pré-operatório de insuficiência mitral sem estemose associada.

A comprovação objetiva dos achados clínicos pôde ser realizada pela análise de cineventriculografia pós-operatória e estudo manométrico, quando foi obsrvada significativa redução das pressōes em artéria pulmonar e capilar pulmonar sem aparecimento de sinais de estenose mitral. Gradientes transvalvares aórticos em pacientes submetidos a implante de anel de Carpentier têm sido descritos ${ }^{12}$, com baixa incidência, o que foi observado em dois pacientes de nosso estudo. Nestes, o gradiente transvalvar aórtico obtido năo foi superior a $20 \mathrm{~mm} / \mathrm{Hg}$.

Portanto, crianças com insuficiência valvar mitral têm sido beneficiadas com as cirurgias reparadoras, sendo que o melhor domínio da tática operatória pela equipe cirúrgica fez com que maior percentagem de pacientes pudesse ser tratada com técnicas reparadoras ( $86 \%$ nos últimos dois anos), evitando, assim, substituiçōes valvares.

Os bons resultados de nossa casuística levaram-nos a adotar as técnias reparadoras da valva mitral como cirurgia de excelência, principalmente em pacientes jovens e crianças. Temos empregado a anuloplastia com anel Gregori-IMC em substituição ao anel de Carpentier, desde outubro de 1987, pelas vantagens de se poder atuar sobre o aparelho subvalvar após sua implantação e por permitir o crescimento normal do anel mitral quando empregado em crianças.

GREGORI Jr., F.; SILVA, S. S.; MOURE, O.; TAKEDA, R.; FAÇANHA, L.; RIBEIRO, I.; GÓIS, L.; SIQUEIRA, J.; GREGORI, T. F.; LOPES, A. G.; KRELING, P.; MIGUITA, L. C.; CORDEIRO, C. - Surgical treatment of mitral insufficiency in children: ten years of reparative techniques. Rev. Bras. Cir. Cardiovasc., 4(3): 202-209, 1989.

ABSTRACT: From April 1979 through January 1989, 71 patients with mitral insufficiency were surgically treated, 53 of them $(74,6 \%)$ submitted to valvuloplasty. From this group 21 patients had associated mitral estenosis. Rheumatic fever was the major cause of the mitral lesions $(79,2 \%)$. All patients, but 3 , were situated in funcional class III and IV (NYHA). Anuloplasty (Carpentier ring and Gregori-IMC) were made associated to plastic procedures at the leaflet and the subvalvar set. In the postoperative period, we observed: no hospital mortality; early postoperative evolution was better in patients subjected to surgery in the period from 84 through 89 , in comparison to the anterior period (79-83); reoperation index very acceptable at the late postoperative $(5,7 \%)$; satisfactory clinical and hemodynamic late evolution; better evolution in the group of patients in the functional class postoperative II and III; better evolution in the group of patients with mitral insufficiency without associated stenosis.

DESCRIPTORS: heart valves, mitral, surgery, children. 
GREGORI Jr. F.; SILVA, S. S.; MOURE, O.; TAKEDA, R.; FAÇANHA, L.; RIBEIRO, I.; GÓIS, L.; SIQUEIRA, J.; GREGORI, T. F.; LOPES, A. G.; KRELING, P.; MIGUITA, L. C.; CORDEIRO, C. - Tratamento cirúrgico da insuficiência mitral em crianças: dez anos de técnicas reparadoras. Rev. Bras. Cir. Cardiovasc., 4(3): 202-209, 1989.

\section{REFERÊNCIAS BIBLIOGRÁFICAS}

1 ANTUNES, M. J.; MAGALHĀES, M. P.; COLSEN, P. R.; KINSLEY, R. H. - Valvuloplasty for rheumatic mitral valve disease: surgical chalenge. J. Thorac. Cardiovasc. Surg., 94: 44-56, 1987.

2 ANTUNES, M. J. \& KINSLEY, R. H. - Mitral valve anuloplasty: results in an underdeveloped population. Thorax, 38: 730-736, 1983.

3 BROFMAN, P. R. - Cirurgia conservadora da valva mitral em pacientes jovens: técnica cirúrgica e resultados. São Paulo, 1986. [Tese. Doutorado. Faculdade de Medicina da Universidade de Sâo Paulo].

4 CARPENTIER, A. - A new reconstructive operation for correction of mitral and tricuspid insufficiency. J. Thorac. Cardiovasc. Surg., 86: 323-337, 1971.

5 CARPENTIER, A.; CHAUVAUD, S.; FABIANI, J. M.; DELOCHE, A.; RELLAND, J.; LESSANA, D.; D'ALLAINES, C.; BLONDEAU, P.; PIWNICA, A.; DUBOST, C. Reconstructive surgery of mitral valve incompetence: ten-year appraisal. J. Thorac. Cardiovasc. Surg., 79: 338-348, 1980.

6 GREGORI Jr., F.; SILVA, S. S.; BABA, K.; QUEIROZ, L. T.; TAKEDA, R.; FAÇANHA, L. A.; SHIGUERU, S.; CANESIN, O. - Um novo modelo de anel protético para pacientes com insuficiência valvar mitral: relato de dois casos. Arq. Bras Cardiol., 50:417-420, 1988.

7 GREGORI Jr., F.; TAKEDA, R.; SILVA, S. S.; FAÇANHA, L.; MEIER, M. - A new technique for repair of mitral insufficiency caused by ruptured chordae of the anterior leaflet. J. Thorac. Cardiovasc. Surg., 96: 765-768, 1988.

8 GREGORI Jr., F.; TAKEDA, R.; SILVA, S. S.; FAÇANHA, L. A.; RIBEIRO, I. A.; AQUINO, W. F.; SHIGUERU, S.; GÓIS, L. E.; SIQUEIRA, J. E.; KRELING, P. A.; GOULART, M. P.; FABIANI, M.; FURLAN, A. I.; MIGUITA, L. C.; CANESIN, O. - Sete anos de experiência com plastia de valva mitral. Arq. Bras. Card., 47: 269-274, 1986.

9 RIBEIRO, E. J.; ALMEIDA, R. M.; FERREIRA, M. J. A.; TENÓRIO, S. B.; CARVALHO, R. G.; BROFMAN, P. R.; LOURES, D. R. R. - Cirurgia reconstrutiva da valva mitral. Arq. Bras. Cardiol., 41: 28, 1983. (Resumo).

10 SAND, M. E. \& NAFTEL, D. C. - A comparison of repair and replacement for mitral valve incompetence. $J$. Thorac. Cardiovasc. Surg., 94: 208-219, 1987.

11 SAUAIA, M. - Curva atuarial. Arq. Bras. Cardiol., 42: 83-93, 1984

12 SCHIAVONE, W. A.; COSGROVE, D. M.; LEVER, H. M.; STEWART, W. J.; SALCEDO, E. E. - Longterm followup of patients with leaflet ventricular outflow tract obs- truction after Carpentier ring mitral valvuloplasty. Circulation, 78 (Supl. I): 60-69, 1986.

13 WOOLER, G. H.; NIXON, P. F. G.; GRIMS, V. A. - Experience with repair of the mitral valve incompetence. Thorax, 37: 49-58, 1982.

\section{Discussão}

\section{DR. PAULO ROBERTO ÉVORA Ribeirão Preto, $S P$}

Após cerca de duas décadas da sua introdução, a cirurgia reparadora da valva mitral tem aumentado, no tratamento da sua insuficiência. Os argumentos favorecendo a troca valvar sobre as correçōes plásticas baseiam-se na dificuldade de aquisição e aperfeiçoamento em um complexo de técnicas, além da suspeita sobre ، a duração destas cirurgias a médio e longo prazo. Alguns autores, como Bashour, Galloway e o próprio Carpentier, apresentam a elitista opinião, com a qual não concordamos, de que a prática da cirurgia conservadora da insuficiência mitral deva ser restrita a poucos centros. De qualquer forma, se este argumento é válido, o Serviço de Cirurgia Cardíaca de Londrina é, sem dúvida, um centro de referência neste campo. Por este motivo, eu e o Hospital do Coração de Ribeirão Preto, instituição que represento, sentimo-nos muito honrados e orgulhosos pela lembrança para ser eu um dos comentadores oficiais deste trabalho. Além deste, outras publicaçōes nacionais e internacionais atestam a criatividade e a importância do Dr. Gregori neste setor da cirurgia cardiaca. Meus agradecimentos à Comissāo Organizadora do 16: Congresso Nacional de Cirurgia Cardíaca. O grupo de Londrina apresenta uma experiência em que 53 pacientes com idade entre 4 e 16 anos $(74,6 \%$ de um total de 73 ) foram submetidos a procedimentos plásticos do aparelho valvar mitral. Destes, em apenas duas crianças não se empregaram anéis rígidos, sendo utilizados 41 anéis de Carpentier e 10 anéis abertos Gregori-IMC associados a outras intervenções sobre as cúspides e aparelho subvalvar. $O$ índice de reoperação nesta série foi de $5,7 \%$ em 10 anos, resultado comparável e, muitas vezes, superior a grandes séries internacionais. (Bachour et alii - Am. Heart J., 113: 1199-1206, 1987). Apenas um paciente faleceu, em uma reoperação com baixissimo índice de mortalidade de 1,9\%. Vinte e cinco pacientes estão operados há mais de 7 anos, atestando a durabilidade dos procedimentos plásticos mitrais. Entre os pacientes submetidos a controle com estudo hemodinãmico, dois pacientes com anel de Carpentier apresentaram gradiente transvalvar aórtico não superior a 20 $\mathrm{mm} / \mathrm{Hg}$, que é um valor desprezível. A obstrução do trato de saída do ventrículo esquerdo tem sido descrita como complicação em cerca de $12 \%$ das valvoplastias com anel de Carpentier em casos de degeneração mixo- 
GREGORI Jr. F.; SILVA, S. S.; MOURE, O.; TAKEDA, R.; FAÇANHA, L.; RIBEIRO, I.; GÓIS, L.; SIQUEIRA, J.; GREGORI, T. F.; LOPES, A. G.; KRELING, P.; MIGUITA, L. C.; CORDEIRO, C. - Tratamento cirúrgico da insuficiência mitral em crianças: dez anos de técnicas reparadoras. Rev. Bras. Cir. Cardiovasc., 4(3): 202-209, 1989.

matosa e nunca em casos de outras etiologias, incluindo as alterações reumáticas mitrais (Schiavone et alii Circulation, 78 (Supl. 2): 60-65, 1986). Uma explicação tem sido estudada pelo próprio grupo do Dr. Carpentier, que atribui este fato, principalmente, ao excessivo estreitamento do ângulo mitro-aórtico e ao excessivo deslocamento da cúspide mitral posterior (Mihaileanu et alii Circulation, 78 (Supl. 2): 78-84, 1986). O anel mitral é uma estrutura dinâmica que se contrai durante a sístole à maneira de um esfincter. Esta função é, compreensivelmente, deficiente em casos de grande dilatação anular ou calcificação. Além disso, tem-se enfatizado que a dilataçāo do anel mitral envolve apenas a sua porção posterior e nunca o anel do folheto aórtico, que é parte do esqueleto fibroso do coração. Estes detalhes anatomofuncionais justificam o emprego dos anéis GregoriIMC, ou outras técnicas de cerclagem do anel posterior sem elementos rígidos, constituindo-se em mais uma crítica aos anéis de Carpentier. No Hospital do Coraçāo de Ribeirão Preto, $45 \%$ dos casos de próteses valvares mitrais, entre 1978 e 1985, eram devidos a lesōes tipo insuficiência. Esta insuficiência diminuiu para $28 \%$ nos anos de 1986 e 1987, caindo para $0 \%$ em 1988. Isto configura uma curva de aprendizado, com a maioria dos pacientes operados pelo Dr. Paulo Ribeiro. A nossa casuística consta de 44 pacientes a partir de 1981, sendo 15 abaixo dos 16 anos de idade. Em 23 pacientes, utilizou-se a técnica descrita por Sauvage, da ampliação do folheto posterior. Quase sempre associamos a cerclagem do anel posterior ao avanço do folheto, ou a realizamos isoladamente, como vimos o Dr. Adib jatene e o Dr. Domongo Braile operar. A estes dois procedimentos, associamos outros detalhes, como: comissurotomias, papilarotomias, encurtamento e transposição de cordas tendineas e liberação de cordas terciárias, de acordo com os princípios da escola francesa. A cerclagem do anel posterior da valva mitral passou a ser feita com tiras de pericárdio bovino, após a experiência de dois casos de anemia hemolítica, nos quais utilizaram-se tiras de Teflon. Um destes pacientes necessitou de reoperação e o outro mantêm-se com o quadro de anemia hemolítica em regressão com tratamento clínico. Dois casos foram reoperados por endocardite com trocas valvares por próteses metálicas e um óbito. Gostariamos de fazer quatro breves perguntas ao Dr. Gregori: 1) Ocorreram, em sua experiência, casos de endocardite e anemia hemolitica? 2) A progressão da lesão reumática não compromete a evolução dos casos tratados com anéis protéticos? 3) Porque a grande maioria dos pacientes foi aperada com anel de Carpentier, sendo que, nos dois primeiros pacientes, este dispositivo não foi utilizado? 4) Porque não há menção ao seguimento destes pacientes com eco-Dopplercardiografia, que, tenho certeza, estar sendo reslizado pelo menos nos últimos tempos? Finalmente, gostariamos de cumprimentar o Dr. Gregori e equipe, pela sua experiência, seguramente uma das maiores do Brasil, e fazer uma crítica construtiva: para tão grande experiência, achamos a discussão do trabalho pobre, no sentido de que nela não constam impressões pessoais, que seriam de grande utilidade para os leitores da "Revista Brasileira de Cirurgia Cardiovascular".

\section{DR. GREGORI \\ (Encerrando)}

Agradeço as palavras do Dr. Évora, do Serviço de Cirurgia Cardiaca do Hospital do Coração de Ribeirão Preto. Lá já estive, a convite deste importante grupo, e posso atestar a seriedade com que tratam este tipo de doença, cuja incidẽncia se sobrepõe à nossa, por se tratar Ribeirão Preto de uma região muito parecida com a de Londrina. Concordamos com sua observação de que gradientes trasvalvares aorticos com anel de Carpentier dificilmente seriam observados em nossa série, uma vez que são mais freqüentes em pacientes com insuficiência mitral de etiologia degenerativa. um paciente de nossa série apresentou hemólise no pós-operatório tardio e corresponde a um dos casos reoperados para substituição valvar. Outros dois pacientes do nosso Serviço, porém adultos, apresentaram hemólise pós-operatória e, em todos, a insuficiência mitral era importante. Acreditamos, portanto, que a presença de hemólise é relacionada com refluxo mitral significativo com anel não recoberto pelo endocárdio. A progressão da lesão reumática compromete, sim. a evolução dos pacientes operados. Devem ser acompanhados de perto, para que novos surtos de doença reumática nāo ocorram, o que, sem dúvida, compromete os resultados. No entanto, é sabido, pelos estudos de Antunes e colaboradores, na África do Sul, que a cirurgia conservadora da valva mitral, mesmo na fase ativa das doenças, apresenta excelentes resultados, tornando a insuficiência cardiaca mais facilmente controlada, em comparação com os doentes tratados, clinicamente. A grande maioria dos pacientes foi submetida a anuloplastia com próteses de Carpentier porque, no início de nossa experiência, este era o procedimento de escolha. A partir de 1987, passamos a utilizar anéis abertos, partindo do princípio de que estes atingiram os mesmos objetivos dos anéis de Carpentier, sem, no entanto, causarem limitações ao crescimento normal do anel mitral de uma criança. Nos dois primeiros pacientes não empregamos nenhum anel protético para a remodelação do anel mitral, porque ainda não estávamos totalmente convencidos de sua necessidade, uma vez que os objetivos eram alcançados com plicaturas do anel mitral à Kay-Wooler. Com o tempo, fomos observando, no nosso material e na literatura, que estava certa a afirmativa de que Carpentier teria, definitivamente, estabelecido as bases anatomopatológicas de lesão valvar mitral e, com suas técnicas, especialmente o anel protético, definitivo a maneira mais correta de tratar esta doença. O nosso intuito com a introdução do anel aberto foi o de manter as funçōes do anel de Carpentier, corri- 
GREGORI Jr. F.; SILVA, S. S.; MOURE, O.; TAKEDA, R.; FAÇANHA, L.; RIBEIRO, I.; GOIS, L.; SIQUEIRA, J.; GREGORI, T. F.; LOPES, A. G.; KRELING, P.; MIGUITA, L. C.; CORDEIRO, C. - Tratamento cirúrgico da insuficiência mitral em crianças: dez anos de técnicas reparadoras. Rev. Bras. Cir. Cardiovasc., 4(3): 202-209, 1989.

gindo mais o desabamento da comissura póstero-mediana pela porçāo retificada do anel protético a este nivel e permitir o crescimento fisiológico do anel mitral pela retirada de sua haste anterior que julgamos, nāo somente desnecessária, como também inconveniente, principalmente em crianças. Atualmente, os pacientes são sempre seguidos, em nosso Serviço, com estudo eco-Dopplercardiográfico. Entretanto parte desta série de pacien- tes não foi estudada no pós-operatório por este método, daí a razão de os pacientes terem sido submetidos a estudo hemodinâmico. Agradeço, mais uma vez, os comentários do Dr. Évora e concordo com a sua crítica sobre pouca exploração na discussão deste trabalho. Pelos trabalhos por mim publicados nestes anos, ele poderá concluir que o capítulo da "Discussão" nunca tem sido o meu forte. 